\title{
Mask-wearing in pediatric age
}

\author{
Susanna Esposito ${ }^{1}$ (D) $\cdot$ Nicola Principi ${ }^{2}$
}

Received: 11 June 2020 /Revised: 11 June 2020 / Accepted: 12 June 2020 / Published online: 30 June 2020

(C) Springer-Verlag GmbH Germany, part of Springer Nature 2020

We thank Jin et al. for the very kind and interesting comment to our paper on the use of masks by children during COVID-19 pandemic [1]. We totally agree that the use of masks in children older than 2 years of age, although not dangerous as in younger subjects, can cause several problems that deserve attention. As highlighted in our article [2], particularly in preschoolers, the use of mask can be obtained only through a strong parental work and, in some cases, compliance is so difficult that it is better that children do not wear the mask and only other preventive measures are implemented. Moreover, masks prepared for adults must be modified, and the method suggested by Jin et al. is one of the measures suggested to adapt the mask size to child face [1].

However, the development of knowledge on COVID-19 has slightly modified the role ascribed to the masks used by children. Initially, it was thought that more than $90 \%$ SARS-CoV-2-infected children were asymptomatic or had a mild disease and that they should wear a mask mainly for preventing transmission of infection to adults, particularly those at the highest risk such as the grandparents [3]. We are now aware that, although with a lower frequency than adults, even children can develop severe COVID-19 and that a not negligible number of cases can present a very severe multisystem inflammatory condition with some features similar to those of Kawasaki disease and toxic shock syndrome [4]. This seems to indicate that children should

Communicated by Peter de Winter

Susanna Esposito

susannamariaroberta.esposito@unipr.it

Nicola Principi

nicola.principi@unimi.it

1 Department of Medicine and Surgery, Pediatric Clinic, Pietro Barilla Children's Hospital, University of Parma, Via Gramsci 14, 43126 Parma, Italy

2 Università degli Studi di Milano, Milan, Italy wear masks also to protect themselves and that more effective masks such as N95 mask specifically prepared for children should be developed. Currently, no international certification standards for the use of these masks on children are available. The current N95 masks available on the market have not been validated in younger children and sizing matters as a too big mask may lead to ineffective use [5].

When N95 masks approved for pediatric age will be available, several children could benefit from using them. Among these, patients with severe chronic underlying disease are at risk of contagion because they are frequently admitted to the hospital for control visits or chronic therapies. However, the real importance of the masks in pediatrics and which type of they should wear in healthy subjects compared to children with severe chronic underlying disease cannot be definitively established until it is clarified what role children have in the spread of the infection. As virus shedding has been found greater in more severe disease [6], it could be supposed that children play a marginal role in the diffusion of the infection. However, transmission of SARS-CoV-2 from asymptomatic subjects has been described [7]. Moreover, even if asymptomatic children shed much less virus than symptomatic patients, this does not exclude that they can infect healthy subjects. The minimum viral load for infection transmission is not precisely defined. Further studies in this regard are urgently needed to solve the problem.

Authors' contributions S.E. and N.P. co-wrote the manuscript and both authors approved the text.

\section{Compliance with ethical statements}

Conflict of interest The authors declare that they have no conflict of interest.

Ethical approval This article does not contain any studies with human participants or animals performed by any of the authors.

Informed consent Not applicable. 


\section{References}

1. Jin K, Min J, Jin X (2020) Re: Esposito et al.: To mask or not to mask children to overcome COVID-19. Eur J Pediatr. https://doi.org/10. 1007/s00431-020-03720-6

2. Esposito S, Principi N (2020) To mask or not to mask children to overcome COVID-19. Eur J Pediatr 9:1-4. https://doi.org/10.1007/ s00431-020-03674-9

3. Lu X, Zhang L, Du H, Zhang J, Li YY, Qu J et al (2020) SARS-CoV2 infection in children. N Engl J Med 382:1663-1665

4. Whittaker E, Bamford A, Kenny J, Kaforou M, Jones CE, Shah P et al (2020) Clinical characteristics of 58 children with a pediatric inflammatory multisystem syndrome temporally associated with SARS-CoV-2 [published online ahead of print, 2020 Jun 8]. JAMA. https://doi.org/10.1001/jama.2020.10369
5. US Food and Drug Administration. N95 respirators, surgical masks, and face masks. Available at: https://www.fda.gov/medical-devices/ personal-protective-equipment-infection-control/n95-respiratorssurgical-masks-and-face-masks\#s3 Accessed on: June 8, 2020

6. Liu Y, Yan LM, Wan L, Xiang TX, Le A, Liu JM et al (2020) Viral dynamics in mild and severe cases of COVID-19. Lancet Infect Dis 20:656-657

7. Furukawa NW, Brooks JT, Sobel J (2020) Evidence supporting transmission of severe acute respiratory syndrome coronavirus 2 while presymptomatic or asymptomatic. Emerg Infect Dis. https:// doi.org/10.3201/eid2607.201595

Publisher's note Springer Nature remains neutral with regard to jurisdictional claims in published maps and institutional affiliations. 